\title{
Loss of heterozygosity in tuberous sclerosis hamartomas
}

Tiina Sepp, John R W Yates, Andrew J Green

\begin{abstract}
We have previously described in tuberous sclerosis (TSC) hamartomas the phenomenon of loss of heterozygosity (LOH) for DNA markers in the region of both the TSC2 gene on chromosome 16 p13.3 and the TSC1 gene on 9q34.
\end{abstract}

We now describe the spectrum of $\mathrm{LOH}$ in 51 TSC hamartomas from 34 cases of TSC. DNA was extracted from leucocytes or normal paraffin embedded tissue, and from frozen paraffin embedded hamartoma tissue from the same patient. The samples were analysed for 11 markers spanning the TSC1 locus and nine markers spanning the TSC2 locus. Twenty-one of 51 hamartomas showed LOH (41\%). There was significantly more LOH on 16 p13.3, with 16 hamartomas showing $\mathrm{LOH}$ around TSC2, and five in the vicinity of TSC1. No hamartoma showed LOH for markers around both loci. All the areas of LOH on chromosome 9 were large, but the smallest region of overlap lay between the markers D9S149 and D9S114, providing independent evidence for the localisation of the TSC1 gene.

These data show that $\mathrm{LOH}$ is a common finding in a wide range of hamartomas, affecting the same TSC locus in different lesions from the same patient but not affecting both loci. These data support the hypothesis that both the TSC genes act as tumour suppressors and that the manifestations of TSC in patients with germline TSC mutations rise from "second hit" somatic mutations inactivating the remaining normal copy of the TSC gene.

(f Med Genet 1996;33:962-964)

Key words: tuberous sclerosis; loss of heterozygosity; hamartoma.

Tuberous sclerosis (TSC) is an autosomal dominant condition characterised by tumourlike malformations (hamartomas) of the skin, brain, heart, kidney, and other organs. The disease prevalence is estimated at 1 in 10000 , with two thirds of cases being sporadic and representing new dominant mutations. Common hamartomas occurring in tuberous sclerosis include cortical tubers, subependymal nodules, retinal astrocytomas, renal an- giomyolipomas, benign giant cell astrocytomas, and cardiac rhabdomyomas.

Two loci have been confirmed for TSC, with approximately $50 \%$ of familial cases linked to TSC1 at $9 \mathrm{q} 34$ and the remainder to TSC2 at 16p13.3. ${ }^{1}$ The TSC1 gene lies in a $2.7 \mathrm{Mb}$ region between markers D9S 149 and D9S $114 .^{2}$ The TSC2 gene has been cloned and about $5 \%$ of patients show large germline deletions of this gene. ${ }^{3}$ The predicted protein sequence has a short area of homology to a RAP1GTPase activating protein (raplGAP or GAP3), and the expressed TSC2 protein has rap1GAP activity in vitro. ${ }^{4}$

We and others have described the phenomenon of loss of heterozygosity $(\mathrm{LOH})$ in the region of the TSC1 or TSC 2 genes in hamartomas from cases of TSC. ${ }^{5-7}$ The presence of LOH on $16 \mathrm{p} 13.3$ in TSC hamartomas, and the finding of large germline deletions in the TSC 2 gene in cases of TSC strongly suggest that the TSC2 gene acts as a tumour or growth suppressor, according to Knudson's hypothesis. ${ }^{8}$ The finding of LOH in TSC hamartomas for $9 \mathrm{q} 34$ suggests a similar role for the TSC1 gene. We now present our findings in a large series of TSC hamartomas.

We analysed 51 TSC hamartomas from 34 confirmed cases of TSC for LOH for markers on $9 \mathrm{q} 34$ and 16p13.3. The hamartomas consisted of 17 renal angiomyolipomas (AML), nine giant cell astrocytomas (GCA), eight fibromas, five cortical tubers, four cardiac rhabdomyomas, four renal cell carcinomas, one shagreen patch, one forehead plaque, one cutaneous keratoacanthoma, and one specimen of diffuse pulmonary lymphangioleiomyomatosis. Data on 13 of these samples have already been reported. ${ }^{56}$ Eleven of the 34 cases were familial, seven linked to the TSC1 gene, two to the TSC2 gene, and two from families too small to be allocated to one or other locus. The samples and normal DNA from the same case were analysed for 11 markers spanning the TSC1 locus and nine markers spanning the TSC2 locus. The markers on chromosome 9 were ASS, D9S64, D9S149, D9S1198, D9S150, ABO, DBH, D9S122, D9S298, D9S114, D9S67, and on chromosome 16 were D16S291, D16S665, KG8, a coding EcoRV polymorphism in exon 40 of the TSC2 gene, D16S525, D16S309, D16S85, and HBAP1. 


\begin{tabular}{|c|c|c|c|c|c|c|c|c|c|c|}
\hline & & & & TS & & & & & & \\
\hline S64 & $S 149$ & $\mathbf{S 1 1 9 8}$ & $A B O$ & S150 & DBH & S122 & S66 & S114 & S298 & S67 \\
\hline
\end{tabular}

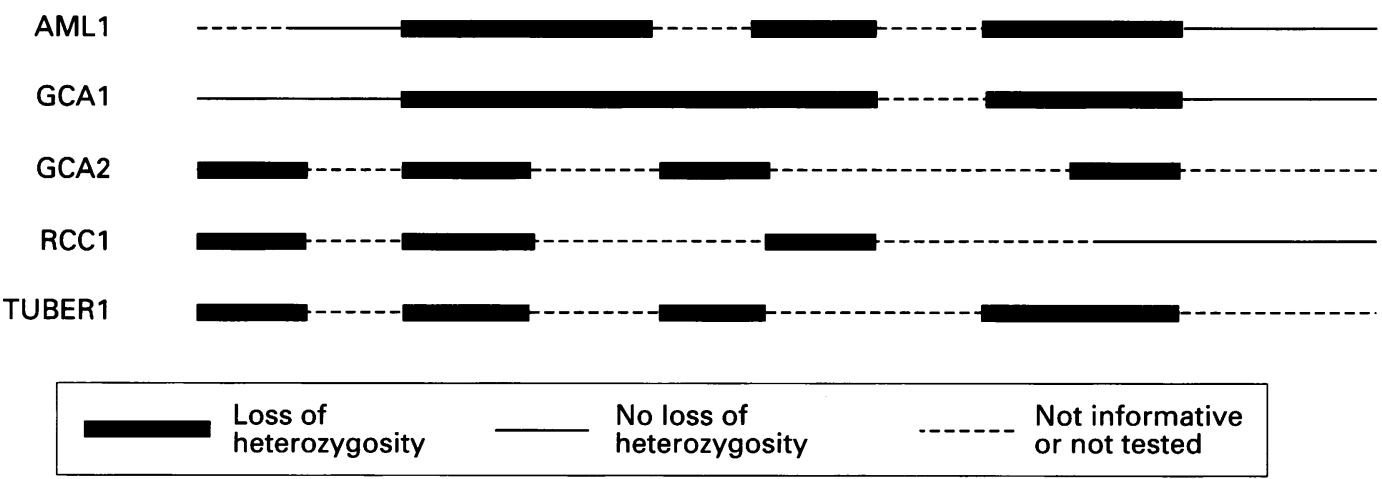

Figure 1 Map of loss of heterozygosity for markers on chromosome $9 q 34$ in TSC hamartomas. The marker order is centromeric to telomeric from left to right. $A M L=$ renal angiomyolipoma, $G C A=$ giant cell astrocytoma, $R C C=$ renal cell carcinoma.

Twenty-one of 51 hamartomas showed LOH (41\%) either in the region of the TSC1 locus or the TSC2 gene. No hamartoma showed $\mathrm{LOH}$ for markers around both loci. Five lesions showed LOH for markers around TSC1 (fig

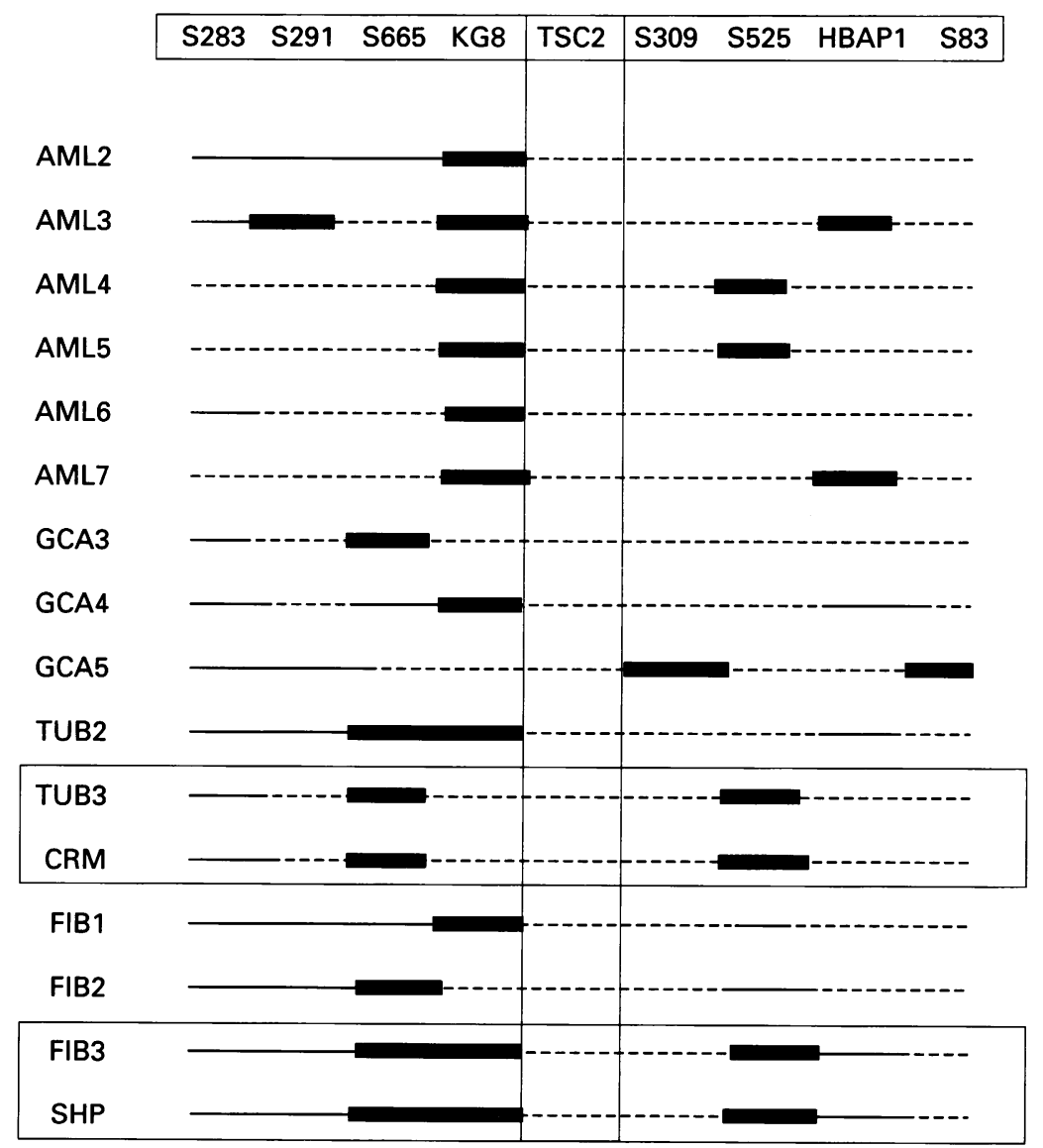

\begin{tabular}{|lll|}
\hline $\begin{array}{l}\text { Loss of } \\
\text { heterozygosity }\end{array}---\begin{array}{l}\text { Not informative } \\
\text { or not tested }\end{array}$ & $\begin{array}{l}\text { No loss of } \\
\text { heterozygosity }\end{array}$ \\
\hline
\end{tabular}

Figure 2 Map of loss of heterozygosity for markers on chromosome 16p13.3 in TSC hamartomas. The marker order is centromeric to telomeric from left to right. The two boxes connect hamartomas which both come from the same case of TSC. AML = renal angiomyolipoma, GCA = giant cell astrocytoma, $R C C=$ renal cell carcinoma, TUB= tuber, $C R M=$ cardiac rhabdomyoma, FIB = fibroma, $S H P=$ shagreen patch.
1). The smallest area of $\mathrm{LOH}$ extended between the disease flanking markers D9S149 and D9S114, independently confirming the locus for TSC1 as defined by linkage analysis.

Sixteen lesions showed variable $\mathrm{LOH}$ for markers in the region of the TSC2 gene (fig 2). A cortical tuber (TUB2) and cardiac rhabdomyoma (CRM) from the same case showed identical patterns of $\mathrm{LOH}$ and the same allele was lost in both hamartomas. The same phenomenon occurred in a shagreen patch and a cutaneous fibroma (FIB2 and SHP) from another case of TSC. We would interpret this finding cautiously, as the markers used represent discrete points along a genome, and the power of LOH studies is insufficient to map areas of somatic deletion effectively.

There did not appear to be any major differences in the frequency of $\mathrm{LOH}$ between the different types of hamartoma. Seven of 17 renal angiomyolipomas showed $\mathrm{LOH}$ and five of nine giant cell astrocytomas showed LOH. Three of eight fibromas, three of five cortical tubers, a shagreen patch, a cardiac rhabdomyoma, and a renal cell carcinoma showed LOH. There was significantly more $\mathrm{LOH}$ for markers in the region of the TSC 2 gene, with a $\chi^{2}$ of 7.52 and a $p$ value of $<0.007$. This excess of $\mathrm{LOH}$ for $16 \mathrm{p} 13.3$ confirms data from other workers studying TSC hamartomas and sporadic renal angiomyolipomas ${ }^{910}$ and may simply reflect that the TSC1 locus is less well defined, and therefore $\mathrm{LOH}$ for $9 \mathrm{q} 34$ is harder to find.

These data show that $\mathrm{LOH}$ is a common finding, occurring in seven different types of hamartoma, including brain hamartomas. LOH occurs in the same TSC locus in different lesions from the same patient but does not affect both loci. These data support the hypothesis that both the TSC genes act as tumour suppressors, and that the manifestations of TSC in patients with germline TSC mutations arise from "second hit" somatic mutations inactivating the remaining normal copy of the TSC gene. Further LOH studies of TSC hamartomas will still be of benefit, as the finding 
of small areas of $\mathrm{LOH}$ on $9 \mathrm{q} 34$ may help refine the TSC1 interval.

We are grateful to the Tuberous Sclerosis Association (UK) for their support of tuberous sclerosis research in Cambridge. TS is funded by the Tuberous Sclerosis Association (UK). We thank Dr Philip Johnson for help with ABO typing. We are grateful to Dr David Kwiatkowski for providing the prime sequence data for the marker D9S1198. We thank all the clinicians and pathologists who allowed us access to patients and tissues.

1 Povey S, Burley MW, Attwood J, et al. Two loci for tuberous clerosis: one on 9q34 and one on 16p13. Ann Hum Genet 1994;58(pt 2):107-27.

2 Povey S, Armour J, Farndon P, et al. Report and abstracts of the Third International Workshop on Chromosome 9 Cambridge, United Kingdom, 9-11 April, 1994. Ann Hum Genet 1994;58(pt 3):177-250.

3 The European Chromosome 16 Tuberous Sclerosis Consortium. Identification and characterisation of the tuberous sclerosis gene on chromosome 16. Cell 1993;75:1-11.
4 Wienecke R, Konig A, DeClue JE. Identification of tuberin, the tuberous sclerosis-2 product. Tuberin possesses specific Rap1GAP activity. $\mathcal{f}$ Biol Chem 1995;270:16409-14.

5 Green AJ, Smith M, Yates JRW. Loss of heterozygosity on chromosome $16 \mathrm{p} 13.3$ in hamartomas from tuberous sclerosis patients. Nat Genet 1994;6:193-6.

6 Green AJ, Johnson PH, Yates JRW. The tuberous sclerosis gene on chromosome $9 \mathrm{q} 34$ acts

7 Carbonara $C$ Longa L, Grosso E et al. 9q34 loss of hetera erosis astrocytoma suggests a growth suppressor-like activity also for the TSC1 gene.
Hum Mol Genet 1994;3:1829-32.

IHum Mol Genet 1994,3:1829-32.

nudson AGJ. Mutation and cancer: statistical study of retinoblastoma. Proc Natl Acad Sci USA 1971;68:820-3.

9 Henske EP, Neumann HP, Scheithauer BW, Herbst EW, Short MP, Kwiatkowski DJ. Loss of heterozygosity in the tuberous sclerosis (TSC2) region of chromosome band $16 \mathrm{p} 13$ occurs in sporadic as well as TSC-associated renal angiomyolipomas. Genes Chrom Cancer 1995;13:295-8.

10 Carbonara C, Longa L, Grosso E, et al. Apparent preferentia loss of heterozygosity at TSC2 over TSC 1 chromosomal region in tuberous sclerosis hamartomas. Genes Chrom Cancer 1996;15:18-25. 